\title{
Glia set the beat
}

DOI:

10.1038/nrn2224

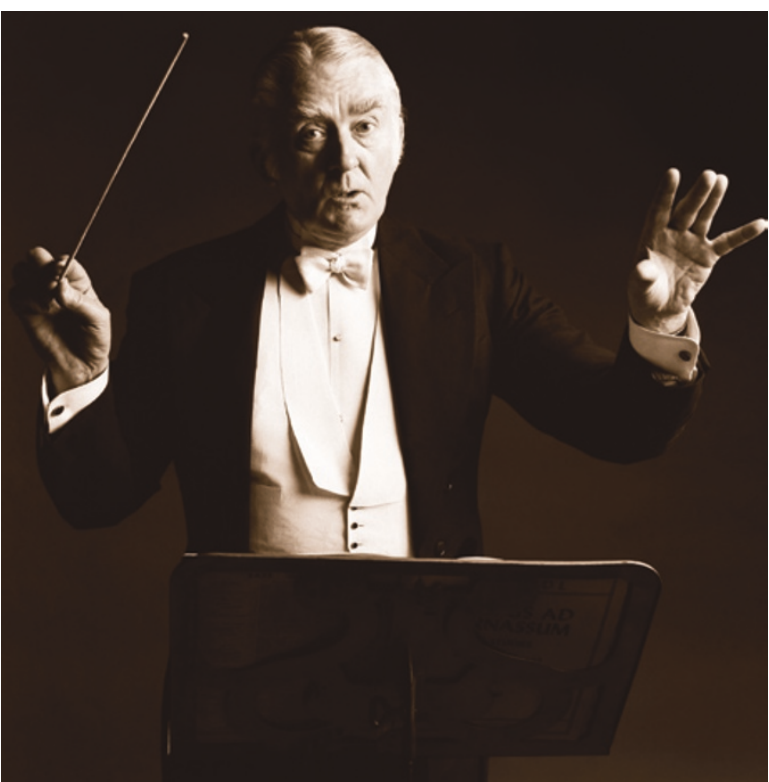

The authors showed that the levels Internal time-keeping mechanisms allow living organisms to adapt their physiological and behavioural processes to the day-night cycle. In Drosphila melanogaster, hundreds of genes that are expressed in adult head tissue exhibit rhythmic daily changes in abundance. Ebony, an enzyme that conjugates $\beta$-alanine to neuromodulators such as dopamine, serotonin and histamine, exhibits robust circadian cycling. Suh and Jackson now show that ebony is expressed in a discrete population of glial cells and acts downstream of genes that regulate the circadian clock to control behavioural rhythmicity in flies.

of ebony peak in the morning, and that ebony RNA cycling is eliminated in flies that carry mutations in the clock genes Period ( $\underline{\text { Per }}$ ) or Timeless (Tim). Ebony mutants exhibited arrhythmic locomotor activity despite the fact that the circadian cycling of Per and Tim were normal, indicating that ebony acts downstream of clock genes to regulate circadian behavioural rhythms.

Whole-mount immunostaining revealed that, in the brain, ebony is localized exclusively to glia. Rhythmic changes in ebony immunofluorescence were observed in regions that are known to contain neurons that control clock function, and co-immunostaining experiments showed that ebony-containing glia are in close proximity to both clock and aminergic neurons, which have previously been shown to have a role in regulating locomotor activity.

Overexpression of ebony in ebony-mutant glia rescued the behavioural phenotype. Furthermore, glial expression of an inactive form of ebony did not rescue the behavioural defects, suggesting a key role for its synthase activity in regulating circadian behaviour.

The authors next examined the regulation of glial ebony by clock cells. They investigated whether ebony is regulated by pigmentdispersing factor ( $\underline{\mathrm{PDF}})$, a neuropeptide that is released from clock cells and has been shown to regulate circadian locomotor activity. However, no differences in the temporal expression pattern of ebony were found in flies that lacked PDF. It is possible that ebony expression is regulated cell-autonomously or by another, as yet undetermined, factor that is released from nearby clock cells.

Finally, Suh and Jackson examined whether altered dopaminergic signalling was related to the arrhythmicity of ebony mutant flies. They found that the hyperactive phenotype of flies that had elevated synaptic dopamine levels (due to a mutation in the dopamine transporter gene DAT) was suppressed by an ebonynull mutation. These results suggest that ebony functions to regulate dopamine signalling, possibly through the rhythmic production of $N$ - $\beta$-alanine dopamine.

This paper suggests that ebony exerts its effects on locomotor activity indirectly, via the modulation of dopamine neurotransmission. Although previous studies have implicated glia in the regulation of activity rhythms, this is the first to shed light on the mechanism through which a particular glial factor can control behavioural rythmicity.

\section{Monica Hoyos Flight}

ORIGINAL RESEARCH PAPER Suh, J. \&

Jackson, F. R. Drosophila ebony activity is required in glia for the circadian regulation of locomotor activity. Neuron 55, 337-339 (2007) 